\title{
Images - Urinary tract reconstruction following ureteral coil embolization for ureterovaginal fistula in a young female patient
}

\author{
Jas Singh, MD; Thomas G. Smith III, MD; O. Lenaine Westney, MD \\ University of Texas MD Anderson Cancer Center, Houston, TX, United States
}

Cite as: Singh J, Smith III TG, Westney OL. Images - Urinary tract reconstruction following ureteral coil embolization for ureterovaginal fistula in a young female patient. Can Urol Assoc J 2022;16(1):E54-6. http://dx.doi.org/10.5489/cuaj.7223

Published online August 26, 2021

\section{Introduction}

The development of a urogenital fistula following treatment for metastatic cervical cancer with chemoradiation is a wellestablished complication. Radiation therapy may induce fistula formation through the process of radiation-induced endarteritis obliterans causing microvascular damage, followed by tissue hypoxia, inflammation, and fibrosis. ${ }^{1}$ While vesicovaginal and enterovaginal fistulae are more commonly encountered, ureterovaginal fistula is a potentially devastating complication due to the associated impacts on renal drainage and function. Here, we present the case of a young female patient who initially underwent an obstructive interventional radiology procedure necessitating reconstructive surgery to re-establish quality of life.

\section{Case report}

A 30-year-old female with a history of stage 1B2 metastatic squamous cell carcinoma of the cervix had undergone management with laparotomy, lymph node sampling, and adjuvant chemoradiation therapy in the periphery. A hysterectomy was not performed during the initial surgery. She then developed persistent postoperative leakage of urine per vagina. She underwent evaluation for suspected vesicovaginal fistula and instead was found to have a left-sided ureterovaginal fistula. She was initially managed with percutaneous nephrostomy (PCN) tube placement for 1-2 years but developed refractory incontinence. There had been no attempts at percutaneous nephroureteral stent placement in this refractory setting. The patient ultimately presented to our center to establish ongoing cancer care.
Following initial consultation with gynecologic oncology, she was referred to interventional radiology in consultation for management, and the decision was made to attempt fistula embolization with endoureteral coil placement. Urology was not consulted. While the persistent incontinence improved, she subsequently developed chronic left flank pain from the PCN and developed recurrent episodes of cystitis and pyelonephritis. Following several years of $\mathrm{PCN}$-based management by her primary team, the patient was referred for urological consultation to review definitive management for her chronic indwelling PCN and to achieve relief of flank pain and recurrent infections. She denied

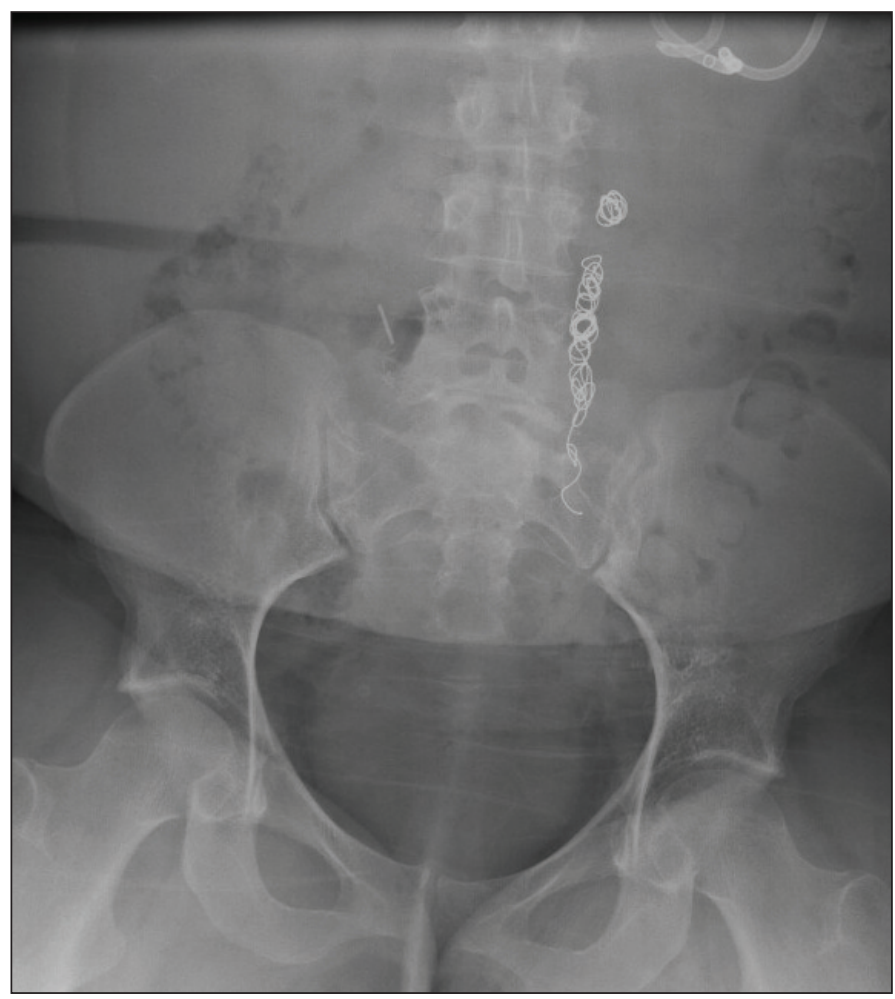

Fig. 1. Plain-film radiograph depicting left-sided endoureteral coils causing obstruction. A left-sided percutaneous nephrostomy tube can be seen at the upper aspect of the image providing urinary drainage for this obstructed collecting system. 


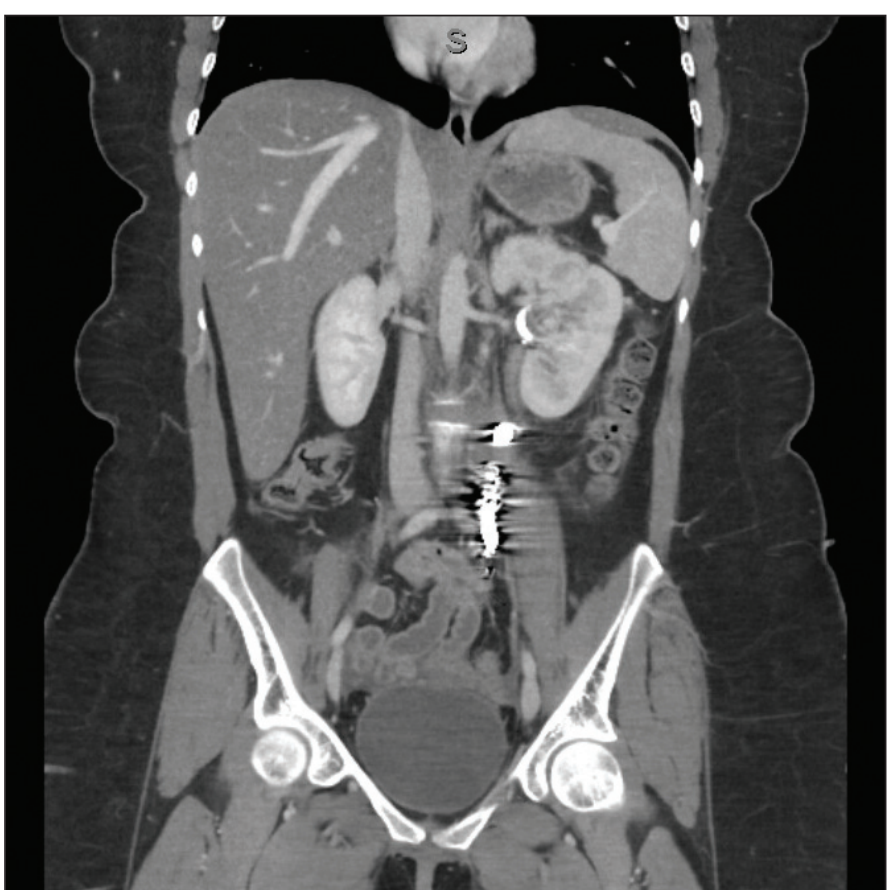

Fig. 2. A coronal view computed tomography scan image demonstrating the same hyperattenuating endoureteral coils with surrounding scatter artifact.

any symptoms related to voiding dysfunction. Radiological imaging demonstrated a long ureteral segment with endoureteral coils causing complete obstruction (Figs. 1, 2). At the time of urological evaluation, the patient continued to be negative for evidence of malignancy since 2015. Renal function was normal (serum creatinine $[\mathrm{SCr}] 59.2 \mathrm{umol} / \mathrm{L}$; estimated glomerular filtration rate [eGFR] 136).

Operative planning with cystoscopy and fluoroscopic urodynamics studies revealed mild hypervascularity consistent with post-radiation cystitis, as well as a reduced bladder capacity of $260 \mathrm{ml}$, with moderately reduced bladder compliance. Given these findings, along with the length and location of the endoureteral coils, the patient was counselled regarding reconstruction with a left-sided ileal ureteral substitution with concomitant augmentation ileocystoplasty. The patient underwent an uneventful reconstructive surgery. Approximately $35 \mathrm{~cm}$ of distal ileum was isolated and used for the repair as a continuous segment with the ileocystoplasty. The diseased ureteral segment was not excised given its fixation to the retroperitoneum from the surrounding fibrosis. The obstructing ureteral coils were identified embedded in the surrounding tissue. The distal $15 \mathrm{~cm}$ of the isolated segment was opened along the antimesenteric border and re-configured into a patch for placement of the ileocystoplasty.

Postoperatively, the patient did well and tolerated a trial of PCN clamping well. She was discharged home with an indwelling double-J ureteral stent and Foley catheter. Postoperative cystogram was performed at three weeks,

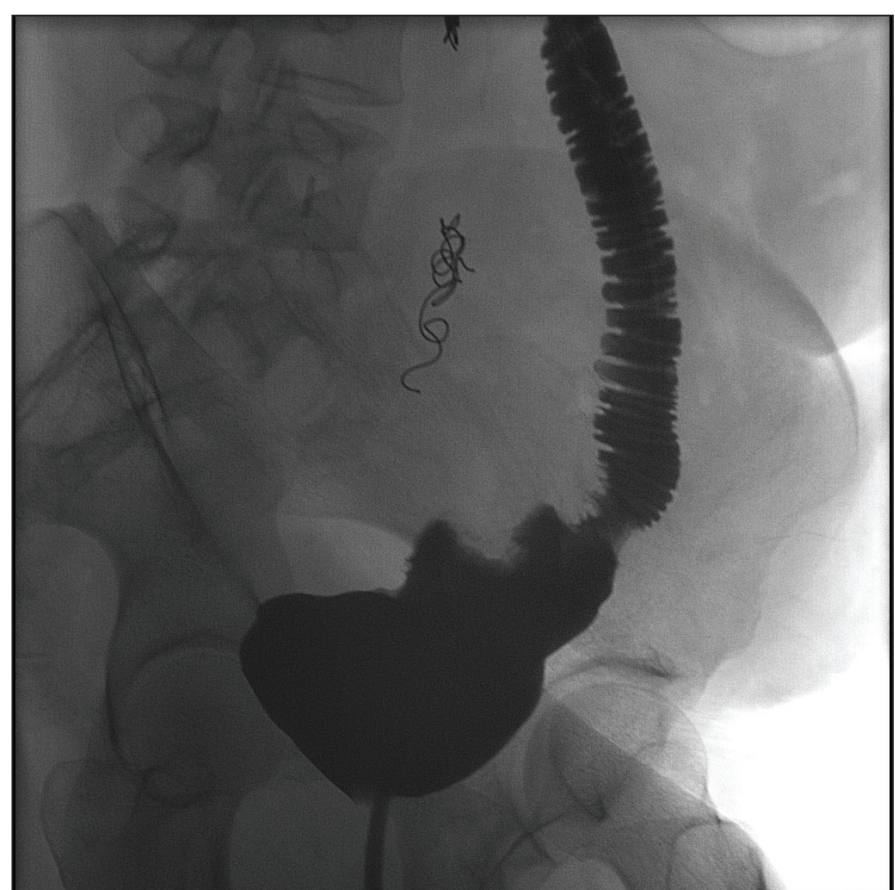

Fig. 3. A postoperative fluoroscopic cystogram demonstrating contrast opacification of the bladder, augmentation ileocystoplasty, and ileal ureteral substitution. The obstructing coils remain in situ, with urinary diversion bypassing the point of obstruction.

which demonstrated normal healing of the bladder with no evidence of leak (Figs. 3, 4). She was then transitioned to clean intermittent catheterization. The ureteral stent was removed postoperatively at six weeks and followup at nine weeks demonstrated normal renal function, with no evidence of hydronephrosis on renal ultrasound. Most recently, at five months' followup, she continues to do very well and denies any recurrent pain or infectious symptoms. Her most recent renal function remains stable (SCr 62.8 umol/L; eGFR 132 ) and renal ultrasound is negative for hydronephrosis.

\section{Discussion}

Percutaneous endoureteral coil embolization for ureterovaginal fistula has been described in the literature for the management of refractory complex lower urinary tract fistulas in patients with persistent urinary leakage despite surgical urinary diversion, poor performance status, limited life expectancy, or as a temporizing measure prior to definitive surgical management. ${ }^{2-4}$ As well, its use as palliation for urinary incontinence in patients with advanced pelvic malignancy has been described with excellent outcomes. ${ }^{5}$ The use of this embolization procedure outside of these parameters is not well-described, given the lack of indications beyond symptom palliation.

In our case, the young patient underwent ureteral embolization with no definitive management plan and without initial urological consultation and recommendation for 


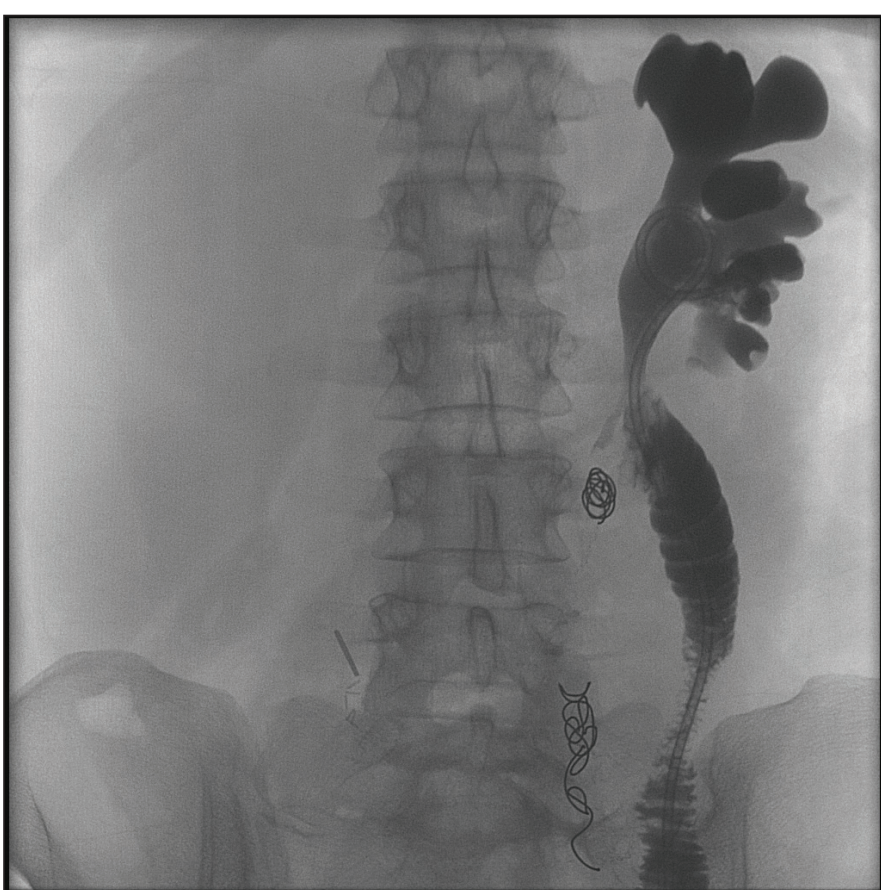

Fig. 4. The proximal aspect of the ileal ureteral substitution can be seen with anastomosis to the left renal pelvis. The indwelling ureteral stent is visualized.

ureterovaginal fistula treatment. Permanent ureteral embolization with permanent chronic nephrostomy placement is unacceptable as definitive management in a young and otherwise healthy patient with no evidence of disease and a good prognosis. This case highlights the importance of a timely and accurate diagnosis and expedited management of urinary fistulae to minimize patient morbidity. Early urological consultation in this case might have spared this patient a destructive procedure and unnecessary repetitive followup procedures.

\section{Conclusions}

Ureterovaginal fistula embolization with percutaneous endoureteral coil placement is an established palliative option in patients with refractory incontinence who are not candidates for definitive repair. This case description highlights the success of this technique in the management of an uncommon clinical scenario.

Competing interests: Dr. Westney has been a consultant for Boston Scientific. The remaining authors do not report any competing personal or financial interests related to this work.

This paper has been peer-reviewed.

\section{References}

1. Narayanan P, Nobbenhuis $M$, Reynolds $K M$, et al. Fistulas in malignant gynecologic disease: Etiology, imaging, and management. RadioGraphics 2009;29:1073-83. https://doi.org/10.1148/ rg. 294085223

2. Amsellem-Ouazana D, Cornud F, Conquy $S$, et al. Management of a malignant urinary fistula by ureteral embolization with coils. Urology 2006;68:427.e1-3. https://doi.org/10.1016/i.urology.2006.02.013

3. Popuri R, Zuckerman DA. Ureteral interventions. Semin Intervent Radiol 2011;28:392-5. https://doi. org/10.1055/s-0031-1296081

4. Shindel AW, Zhu H, Hovsepian DM, et al. Ureteric embolization with stainless-steel coils for managing refractory lower urinary tract fistula: A 12-year experience. BJUI 2007;99:364-8. https://doi.org/10.1111/j.1464-410X.2006.06569.x

5. Natarajan V, Boucher NR, Meiring $P$, et al. Ureteric embolization: an alternative treatment strategy for urinary fistulae complicating advanced pelvic malignancy. BJUI 2007;99:147-9. https://doi.org/10.1111/i.1464-410X.2006.06522.x

Correspondence: Dr. Jas Singh, University of Texas MD Anderson Cancer Center, Houston, TX, United States; jingh2@mdanderson.org 